\title{
Circadian changes in anticoagulant effect of heparin infused at a constant rate
}

\author{
H A DECOUSUS, M CROZE, F A LEVI, J G JAUBERT, B M PERPOINT, J F DE BONADONA, \\ A REINBERG, P M QUENEAU
}

\begin{abstract}
Six patients with venous thromboembolism were treated with heparin, administered intravenously by a constant infusion pump. The initial daily dose of heparin was adjusted to keep the activated partial thromboplastin time, sampled at 0800 , between 1.5 and 2.5 times the control level. Once that level was obtained, this dose was kept constant. Anticoagulation was thereafter measured, every four hours for $\mathbf{4 8}$ hours, by activated partial thromboplastin time, thrombin time, and coagulation factor $\mathrm{Xa}$ inhibition assay.

The results of all three coagulation tests showed a circadian variation in the six patients. Maximum values were achieved at night and minimum values in the morning. These circadian variations were reproduced for two consecutive days. Differences between night and morning values reached almost $50 \%$ for activated partial thromboplastin time, $60 \%$ for thrombin time, and $40 \%$ for factor $X a$ inhibition assay. This circadian variation resulted from two rhythms, a circadian rhythm lasting 24 hours and an ultradian rhythm lasting 12 hours, which were detected by cosinor analysis for each coagulation test $(p<0.01)$. A circadian $r$ hythm was
\end{abstract}

Department of Internal and Therapeutic Medicine, Hôpital de Bellevue, Saint-Etienne Cédex, France

H A DECOUSUS, MD, assistant professor of internal medicine

B M PERPOINT, MD, assistant professor of internal medicine

J F DE BONADONA, MD, resident in medical science

P M QUENEAU, professor of internal medicine

Blood Transfusion Centre, Hôpital de Bellevue

M CROZE, MD, assistant professor of haematology

J G JAUBERT, MD, assistant professor of haematology

Equipe de Recherches de Chronobiologie Humaine, Fondation Adolphe de Rothschild, 75019 Paris, France

F A LEVI, MD, PHD, chargé de recherche au Centre National de la Recherche Scientifique

A REINBERG, MD, PHD, chargé de recherche au Centre National de la Recherche Scientifique

Correspondence to: Dr H A Decousus. detected individually in most of the patients for each coagulation test $(p<0.05)$. All patients had a nocturnal peak in activated partial thromboplastin time on both days. In four patients this peak exceeded the upper desired limit of activated partial thromboplastin time.

These rhythms should be taken into account when evaluating the dosage of heparin to be administered.

\section{Introduction}

Heparin is the best anticoagulant for the initial treatment of venous thromboembolism.1 ${ }^{2}$ Continuous intravenous administration of heparin was proposed as a means of avoiding the acute variations in heparin anticoagulant effect that occur during discontinuous administration. Even so, in up to $30 \%$ of patients with venous thromboembolism treated with continuous intravenous administration of heparin bleeding complications occur $^{3}$ or thrombosis recurs." At present there is no agreement on whether a coagulation test should be used to adjust heparin dosage. ${ }^{5}$ This suggests that factors other than those previously investigated may influence the effects of heparin. As the time at which a drug is administered often influences its effects ${ }^{\circ}$ we investigated this possibility for heparin administered intravenously by a constant infusion pump. Such a variation might explain some cases of bleeding or recurrence of venous thromboembolism, observed despite continuous intravenous administration of heparin. We studied six patients with venous thromboembolism treated with continuous intravenous administration of heparin. Activated partial thromboplastin time, thrombin time, and coagulation factor $\mathrm{Xa}$ inhibition assay were determined every four hours for $\mathbf{4 8}$ hours.

\section{Patients and methods}

Patients-Six patients entering our clinical study unit between February and April 1984 with the diagnosis of venous thromboembolism (without massive pulmonary embolism) participated in this study. Their informed consent was obtained. Two were men and four women. Their mean age was 62 (range 51-78) years and mean weight 67 (range 48-85) kg. None was a smoker or alcoholic; all had normal renal and hepatic function as assessed by serum creatinine, 
TABLE I-Clinical data on six patients with venous thromboembolism and daily dose of heparin

\begin{tabular}{|c|c|c|c|c|c|c|}
\hline & \multicolumn{6}{|c|}{ Case No } \\
\hline & 1 & 2 & 3 & 4 & 5 & 6 \\
\hline $\begin{array}{l}\text { Age (years) } \\
\text { Sex } \\
\text { Weight (kg) } \\
\text { Venous thromboembolism } \\
\text { Aetiology of venous } \\
\text { thromboembolism } \\
\text { Heparin dose }(\mathrm{U} / \mathbf{k g} / 24 \mathrm{~h})\end{array}$ & $\begin{array}{c}51 \\
F \\
48 \\
\text { Pulmonary embolism } \\
\text { Leg trauma } \\
390\end{array}$ & $\begin{array}{c}78 \\
M \\
65 \\
\text { Pulmonary embolism } \\
\text { Idiopathic } \\
\text { 362 }\end{array}$ & $\begin{array}{c}53 \\
\mathbf{M} \\
66 \\
\text { Pulmonary embolism } \\
\text { Malignancy } \\
\mathbf{5 8 2}\end{array}$ & $\begin{array}{c}64 \\
\text { F } \\
64 \\
\text { Deep vein thrombosis } \\
\text { Idiopathic } \\
247\end{array}$ & $\begin{array}{c}52 \\
F \\
71 \\
\text { Pulmonary embolism } \\
\text { Leg trauma } \\
595\end{array}$ & $\begin{array}{c}72 \\
\begin{array}{c}72 \\
85 \\
\text { Deep vein thrombosis } \\
\text { Malignancy } \\
237\end{array}\end{array}$ \\
\hline
\end{tabular}

During the study antacid preparations were prescribed for cases 2,4 , and 6 , calcium antagonists for case $2, \mathrm{H}_{2}$ antihistamines for cases 4 and 6 , and thiazide diuretics for case 4 .

TABLE II-Circadian rhythms in activated partial thromboplastin time (APTT), thrombin time (TT), and factor Xa inhibition assay (XaI) measured every four hours for 48 hours in six patients receiving continuous intravenous heparin treatment

\begin{tabular}{|c|c|c|c|c|c|}
\hline \multirow{2}{*}{ Coagulation tests } & \multirow{2}{*}{$\begin{array}{c}\text { No of } \\
\text { measurements }\end{array}$} & \multicolumn{3}{|c|}{ Circadian variables } & \multirow{2}{*}{ p values" } \\
\hline & & Mesort (SEM) & Double amplitudeł (2 SEM) & Acrophase\& (2 SEM) & \\
\hline \multirow{2}{*}{$\begin{array}{l}\text { Raw values: } \\
\text { APTT } \\
\text { TT } \\
\text { XaI } \\
\text { Percentage of individual } 24 \text { hour mean: } \\
\text { APTT } \\
\text { TT } \\
\text { XaI }\end{array}$} & $\begin{array}{l}78 \\
78 \\
78\end{array}$ & $\begin{array}{l}53.9(1.4) \mathrm{s} \\
0.32(0.02) \mathrm{U} / \mathrm{ml} \\
0.38 \mathrm{U} / \mathrm{ml}\end{array}$ & $\begin{array}{l}23.8(5.2) \mathrm{s} \\
0.16(0.06) \mathrm{U} / \mathrm{ml} \\
0.11 \mathrm{Ul} / \mathrm{ml}\end{array}$ & $\begin{array}{l}0030(2250,0210) \\
0040(2130,0350) \\
2320\end{array}$ & $\begin{array}{l}0.0001 \\
0.007 \\
0.12\end{array}$ \\
\hline & $\begin{array}{l}78 \\
78 \\
78\end{array}$ & $\begin{array}{l}100(2) \\
100(2) \\
100(2)\end{array}$ & $\begin{array}{l}45(7) \\
48(8) \\
29(6)\end{array}$ & $\begin{array}{l}0030(2320,0140) \\
0100(2340,0220) \\
2340(2200,0120)\end{array}$ & $\begin{array}{l}0.0001 \\
0.0001 \\
0.0001\end{array}$ \\
\hline
\end{tabular}

serum albumin, and bilirubin concentrations and transaminase activities. In all patients the diagnosis of deep venous thrombosis was confirmed by ascending phlebography. Four had a pulmonary embolism confirmed by perfusion lung scanning.

Treatment-All patients received sodium heparin $(5000 \mathrm{U} / \mathrm{ml})$ from the same laboratory (Roche). Each received the same heparin lot during the two day study. Three different infusion pumps were used : two Robomedic 100 (Durillon-Lasseigne, Lyon, France) and one Soma seringe type S (Hyco-Aulas-Gauthier, Dardilly, France). Each patient received heparin by the same pump over the two days. Heparin was diluted in $0.9 \%$ saline up to $50 \mathrm{ml}$ in a plastic syringe (Becton-Dickinson). The syringe was changed every 24 hours at 0800 . The infusion rate $(2 \mathrm{ml} / \mathrm{h})$ was checked every four hours during the two day study. Before the beginning of the study the daily heparin dose was adjusted to keep the activated partial thromboplastin time, sampled at 0800 and measured in fresh plasma, between 1.5 and 2.5 times the control level of $28(42-70)$ seconds. ${ }^{2}$ Once this dose had been determined the patient entered the study and the dose was kept constant. Heparin was injected through a cathlon placed in a forearm vein. During the study no patient received any oral anticoagulant, antiplatelet drug, dextran, or hormonal treatment. All patients were resting in bed and were taking meals at fixed times $(0700,1200$, and 1900$)$.

Coagulation tests-Venous blood was taken at 0800 and then every four hours for $\mathbf{4 8}$ hours with a non-heparinised cathlon placed in the forearm not used for heparin infusion. The samples collected at 0800 were obtained before the daily replacement of the syringe, except once. The first millilitre was discarded, and the next five were collected in polystyrene tubes (nine volumes of blood and one volume of $0 \cdot 129 \mathrm{M}$ trisodium citrate). Normal plasma was pooled and stored at $-80^{\circ} \mathrm{C}$ in $1 \mathrm{ml}$ aliquots. After being collected each blood sample was centrifuged for 10 minutes at $2500 \mathrm{~g}$ and stored frozen at $-80^{\circ} \mathrm{C}$ until assayed. All plasmas were tested within a week after the blood collection immediately after thawing at $37^{\circ} \mathrm{C}$. All samples collected at $\mathbf{0 8 0 0}$ were also assayed on fresh plasma for activated partial thromboplastin time and thrombin time. In one patient activated partial thromboplastin time in fresh plasma was determined at 0800,1200 , and 1600 on the first day and every four hours on the second day. Assaying for activated partial thromboplastin time was performed in duplicate using the same reagent (Auto activated partial thromboplastin time reagent, General Diagnostics Laboratories) and a clot detecting device (Coag-A-Mate X2, General Diagnostics Laboratories). Activated partial thromboplastin time was expressed in seconds. Nermal plasma activated partial thromboplastin time was 28.2 seconds with a coefficient of variation of $1.61 \%$. For high activated partial thrombioplastin times (mean 100.8 seconds) the coefficient of variation was $3 \cdot 18 \%$. Assay for thrombin time, described by $\mathrm{Raby}^{\mathrm{B}}$ was performed with pretitrated thrombin using a handling system. Assay for coagulation factor Xa inhibition was performed with the Coag-A-Mate X2, according to the method of Yin et al.' Clotting times for thrombin time and factor $\mathrm{Xa}$ inhibition assay were converted into heparin activities $(\mathrm{U} / \mathrm{ml}$ of plasma). At 0.3 $\mathrm{U} / \mathrm{ml}$ heparin the coefficients of variation of thrombin time and factor $X a$ inhibition assay were $7.27 \%$ and $4.59 \%$, respectively. The 18 plasma samples taken at 0800 were analysed for activated partial thromboplastin time and thrombin time both when fresh and after storage at $-80^{\circ} \mathrm{C}$. There was a highly significant correlation between the values from both fresh and frozen samples $(r=0.87$ for activated partial thromboplastin time; $r=0.89$ for thrombin time; $p<0.01$ ). Activated partial thromboplastin time, however, was, on average, 15 seconds (24\%) shorter and thrombin time $0.03 \mathrm{U} / \mathrm{ml}(12 \%)$ shorter in the frozen samples than in the fresh ones. When 10 consecutive samples from one patient were analysed for activated partial thromboplastin time, both when fresh and after freezing, similar results were obtained $(r=0.82 ; p<0.01$, mean difference $22 \%)$.

Statistical analysis-Data were analysed by both conventional and cosinor methods.10 The analyses were performed on raw values and data transformed as percentages of the individual 24 hour mean. This transformation reduces interpatient variability. ${ }^{10}$ The cosinor method was used to obtain the best fitting cosine function approximating all data with a given period. A rhythm was characterised by:

(1) its period $(\tau)$-that is, the length of time between successive repetitions-with an ultradian rhythm being one for which $\tau$ is less than 24 hours and a circadian rhythm one for which $\tau$ is approximately equal to 24 hours:

(2) its acrophase, the location of the peak time, for a given $\tau$;

(3) its amplitude, equal to half the difference between peak and trough in a given $\tau$;

(4) its mesor, the rhythm adjusted mean for the $\tau$ under consideration.

A rhythm is statistically validated by an F test of the rejection of null amplitude hypothesis $(p<0.05)$.

\section{Results}

Table I gives clinical details for the six patients studied. During the study one (case 1) sustained mild bleeding (haemoptysis) at the end of the night. Venous thromboembolism did not recur in any patient. The mean daily dose of heparin was $402 \mathrm{U} / \mathrm{kg} / 24 \mathrm{~h}$ (range 237-595 U/kg/24 h). Table I gives the dose for each patient. When compared with the expected daily rate of infusion $(48 \mathrm{ml} / 24 \mathrm{~h})$ the pumps were fast on nine occasions (mean advance $1.9 \%(0.9 \mathrm{ml}$ ) range $1-5 \%)$ and slow on three $(1 \%(0.5 \mathrm{ml})$ for each time). When the rate of infusion was checked every four hours no importan fluctuation or systematic error was detected. 
As figure 1 shows, whether the mean values for the six patients were expressed as raw values or as percentages of the individual 24 hour mean the anticoagulant effect was not constant during the 24 hours. A circadian variation was observed, which was reproducible from one day to the next, with a peak at night and a trough in the morning. A similar pattern was observed with the results of the three coagulation tests. Differences between peak (at 0400) and trough (at 0800) reached almost $50 \%$ for activated partial thromboplastin time, $60 \%$ for thrombin time, and $40 \%$ for coagulation factor $\mathrm{Xa}$ inhibition assay (paired $t$ test: $\mathrm{p}<0.001$ ). Table II gives results obtained with cosinor analysis. A circadian (24 hour) rhythm was detected for activated partial thromboplastin time and thrombin time expressed as raw values. This rhythm was also detected for factor $\mathrm{Xa}$ inhibition assay expressed as a percentage of individual 24 hour mean. Acrophases (peak times) occurred at the beginning of the night for the three tests. The double amplitude (difference between peak and trough) was considerable (almost $50 \%$ ) for activated partial thromboplastin time and thrombin time. The good reproducibility of these rhythms from one day to the next was further validated by cosinor analysis performed on separate data from each study day $(p<0.01$ for each day and for each coagulation test) with similar acrophases.

In addition to the 24 hour rhythm, an ultradian rhythm with a period of 12 hours was found for each coagulation test by the cosinor method ( $p<0.01$ ). The acrophases (peak times) of these ultradian rhythms occurred at 0210 and 1410 for activated partial thromboplastin time, at 0230 and 1430 for thrombin time, and at 0225 and 1425 for factor $\mathrm{Xa}$ inhibition assay. Their double amplitudes were smaller than those seen in the 24 hour rhythms-for example, $20 \%$

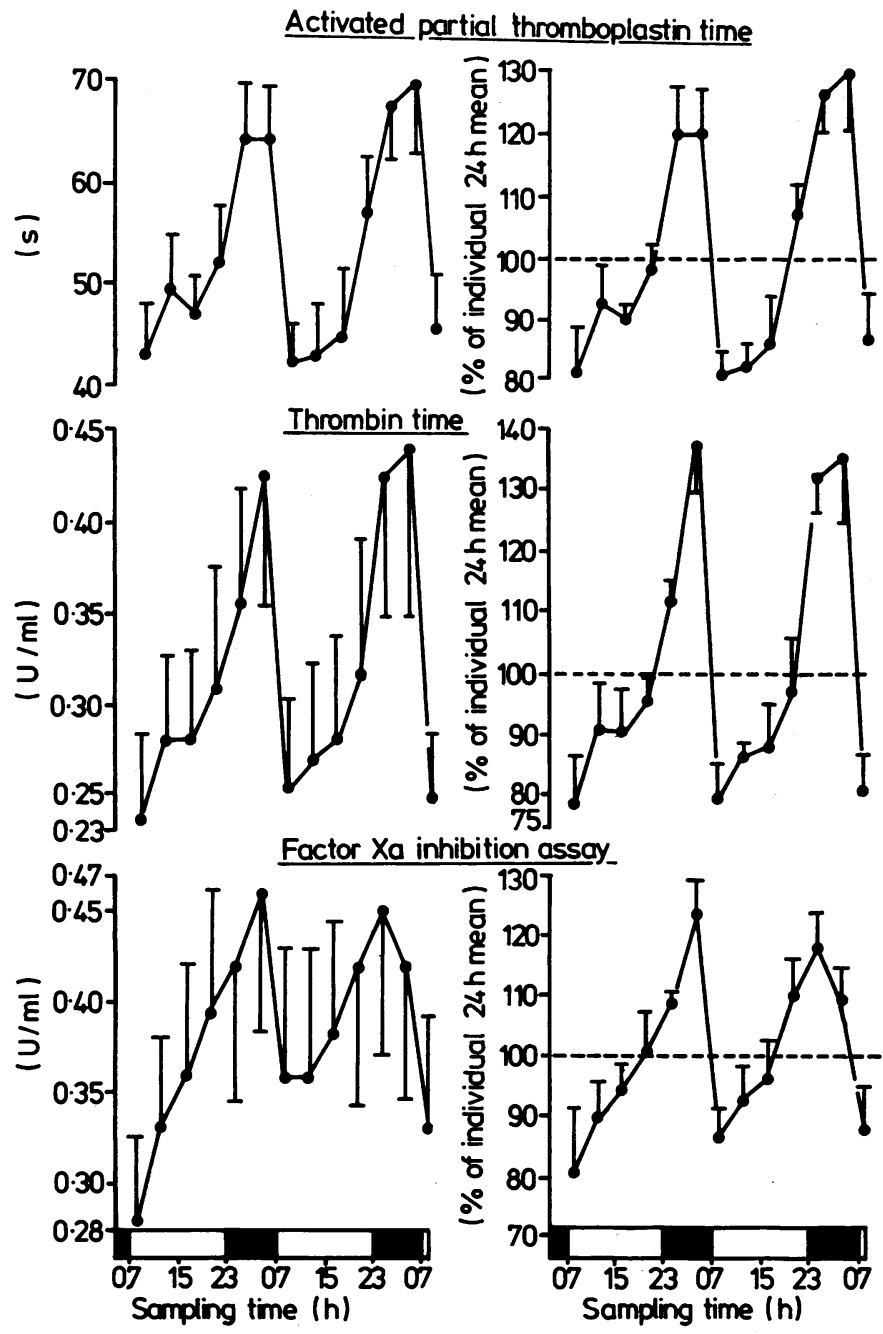

FIG 1-Time course of activated partial thromboplastin time, thrombin time, and factor $\mathrm{Xa}$ inhibition assay measured every four hours for $\mathbf{4 8}$ hours in six patients receiving continuous intravenous heparin treatment. Vertical bars represent mean (SEM). Periods of sleep ( $\square$ ) and wakefulness ( $\square$ ) are indicated. Significant circadian ( 24 hour) and ultradian (12 hour) rhythms were validated by cosinor analysis for the three coagulation tests. for activated partial thromboplastin time, $32 \%$ for thrombin time, and $14 \%$ for factor $\mathrm{Xa}$ inhibition assay. The algebraic sum of these two rhythmic components was computed as Fourrier's transformation for each variable. Figure 2 shows the computed waveform of the circadian variation in activated partial thromboplastin time as an example.

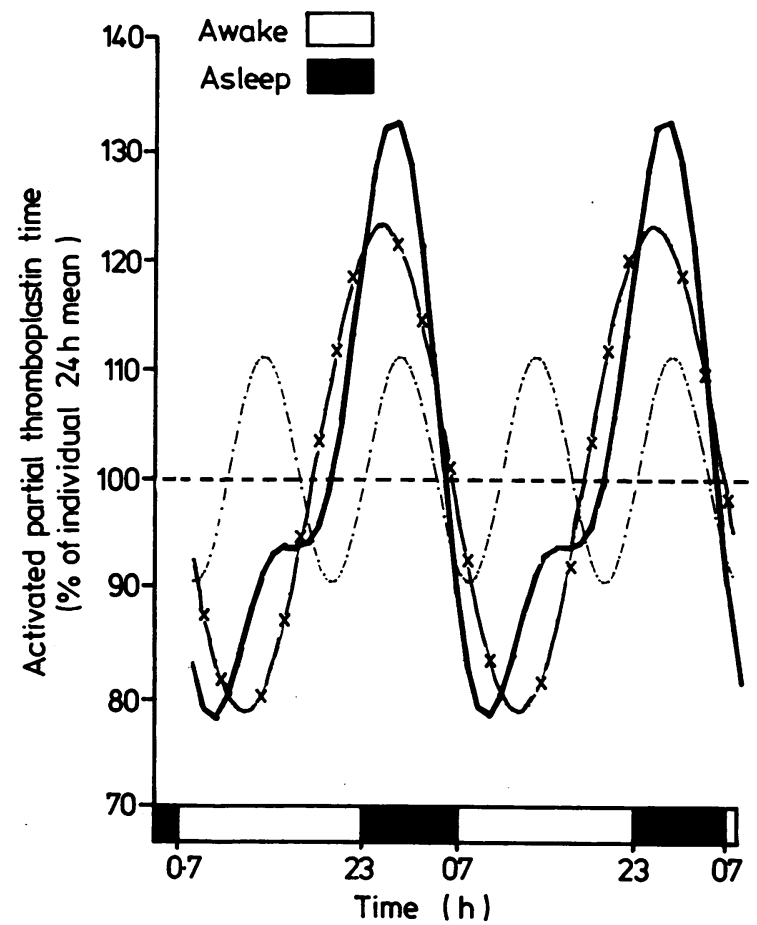

FIG 2-Computed waveform of circadian variation in activated partial thromboplastin time in six patients receiving continuous intravenous heparin treatment. Both a circadian rhythm with a period of 24 hours $(x-x-x)$ and an ultradian rhythm with a period of 12 hours $(-\cdot-\cdot-\cdot)$ were statistically validated by cosinor analysis. Their algebraic sum $\stackrel{\longrightarrow}{\longrightarrow}$ was computed as a Fourrier's transformation.

Figure 3 shows variations in activated partial thromboplastin time in each patient. All patients had a night peak on both days, but the magnitude of the difference between peak and trough values varied from one patient to another. For example, night and morning activated partial thromboplastin time differed by more than 50 seconds in cases 1 and 2 . A significant circadian rhythm $(p<0.05)$ was found by cosinor analysis in four patients (cases $1,2,3$, and 4) for activated partial thromboplastin time and in three patients (cases 1,2 , and 3 ) for thrombin time $(p \simeq 0.08$ in case 5$)$. The circadian rhythm in factor Xa inhibition assay was statistically validated $(p<0.05)$ in four patients (cases 1, 2, 3, and 5). In all patients the acrophase occurred at the beginning of the night. The double amplitude was greatest in cases 1 and 2 for the results of all three coagulation tests. No evident correlation was found between the characteristics of the rhythm (detection or double amplitude, or both) and the clinical data on each patient (for example, heparin dose).

\section{Discussion}

Continuous intravenous administration of heparin did not provide a constant anticoagulant effect in the six patients. Indeed, results of the three coagulation tests showed a circadian variation, with a maximum anticoagulation at night and a minimum in the morning, which was reproducible over two consecutive days (fig 1). Variations between night and morning were considerable, with a mean difference reaching almost $50 \%$ in activated partial thromboplastin time, $60 \%$ in thrombin time, and $40 \%$ in factor $\mathrm{Xa}$ inhibition assay (fig 1 ). This circadian variation resulted from two rhythms, a circadian rhythm lasting 24 hours and an ultradian rhythm lasting 12 hours, 


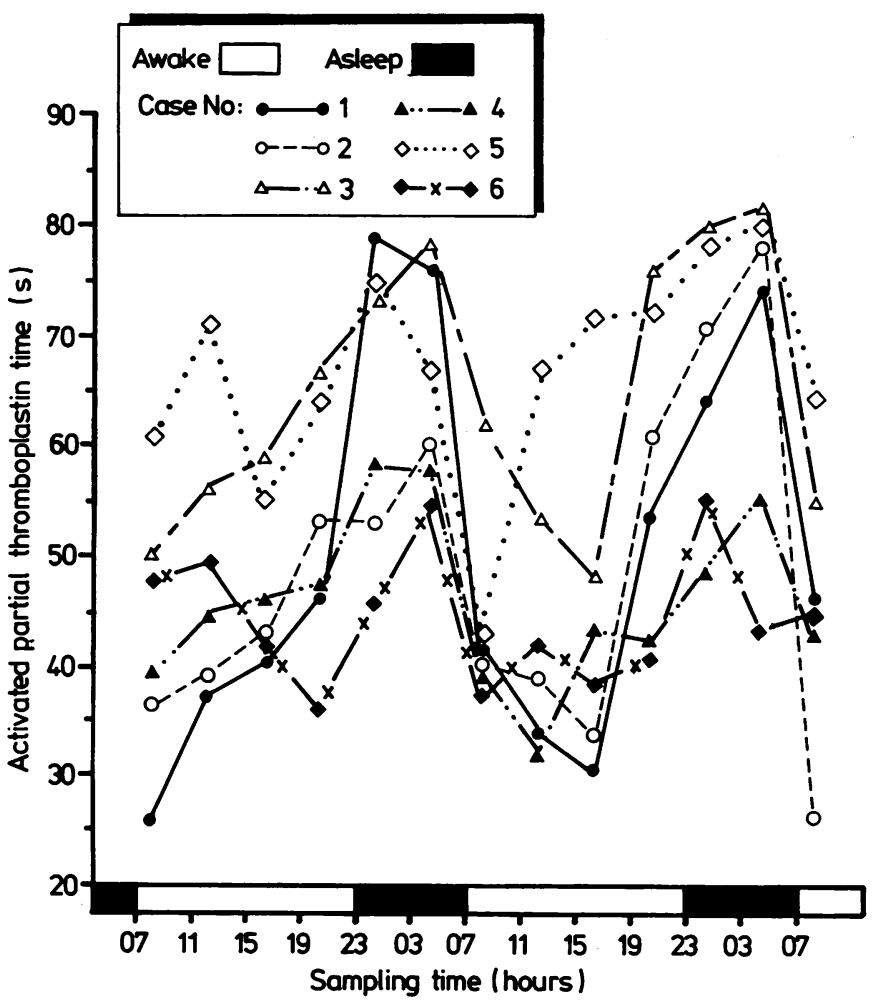

FIG 3-Time course of individual activated partial thromboplastin times measured every four hours for $\mathbf{4 8}$ hours in six patients receiving continuous intravenous heparin treatment. Significant circadian rhythms were validated by cosinor analyses for cases $1,2,3$, and 4 .

detected for each coagulation test. Indeed, the sum of these two rhythms, in activated partial thromboplastin time for example (fig 2), provided a rather good fit of the experimental data (fig 1) and explained the nocturnal peak and the trough in the morning. To our knowledge, this is the first report of a circadian and an ultradian rhythm in the effect of a drug administered by continuous intravenous infusion.

These rhythms cannot result from variations in the rate of infusion as the mean difference between the actual and expected daily rates of infusion was less than $2 \%$. We used activated partial thromboplastin time because it is the test most commonly used to monitor heparin. ${ }^{11}$ The choice of the Auto activated partial thromboplastin time reagent was justified by its good sensitivity to heparin. ${ }^{11}$ Thrombin is also used to monitor heparin, ${ }^{12}$ like factor $\mathrm{Xa}$ inhibition assay particularly for this test since the finding of low molecular weight heparins. ${ }^{13}$ Assays were performed on frozen plasma so that all the samples from the same patient could be run in the same batch of assay. We found a $25 \%$ decrease in activated partial thromboplastin time values in frozen compared with fresh samples but showed that this difference was not influenced by the sampling time. Thus the night peak in activated partial thromboplastin time, measured in a fresh sample, would probably have exceeded the desired upper limit of 70 seconds by even more in four patients (fig 3). As the adjustment of the daily heparin dose was based on activated partial thromboplastin time at 0800 (trough time) a risk of an overdosage of heparin was encountered. If night values of activated partial thromboplastin time had served to guide the choice of the daily heparin dose an underdosage of heparin might have resulted. Some clinical studies have shown a correlation between activated partial thromboplastin time values and the incidence of either bleeding ${ }^{4}$ or recurrence of venous thromboembolism 7 ; the rhythms that we have shown may explain some of these complications of heparin treatment. It is of interest that one patient (case 1), who showed a prominent rhythm in the results of all three coagulation tests, experienced bleeding during heparin treatment.
The cause of these rhythms in the effect of heparin is not known at present. There may be a circadian rhythm in the pharmacokinetics of this agent as has been shown for many drugs. ${ }^{14}$ Alternatively, a recent study in normal volunteers showed a spontaneous circadian variation in coagulation with an increased clotting tendency in the morning, ${ }^{15}$ which would be consistent with the circadian variation of the heparin effect reported here.

We believe that this circadian variation should be taken into account when evaluating the heparin dose to be administered. The time at which blood is taken for coagulation tests appears to be crucial, and the rate of heparin infusion might be adapted according to these biological rhythms. Further studies will be necessary before extrapolating our results to all patients receiving treatment with heparin. Such studies should also investigate the effects of variations in heparin administration on the incidence of bleeding and recurrence of venous thromboembolism.

We thank the nurses of our clinical department and the technicians from the transfusion centre, without whom this study could not have been performed; Professor J C Bertrand, Dr Y Page, and Professor C P Brizard for their collaboration; Mr M Meckouri for his help with the data analysis; and Mrs M C Breas and Mrs E Chastel for typing the manuscript.

\section{References}

1 Barrit DW, Jordan SC. Anticoagulant drugs in the treatment of pulmonary embolism: a concrolled trial. Lancet $1960 ; 1$ i $1309-12$.

Bauer G. Heparin as a therapeutic against thrombosis. Acte Chir Scand 1942; 86:267-72

3 Mant MJ, O'Brien B, Thong KL, et al. Haemorrhagic complications of heparin therapy. Lancet 1977 .

4 Wilson JE, Bynum LJ, Parkey RW. Heparin therapy in venous thromboembolism. Am F.Med 1981;70:808-16.

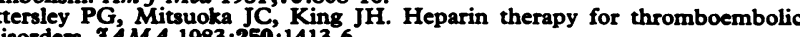
disorders. FAMA 1983;250:1413-6.

1981;34:171-8. , Gallus A, Hirsh J, et al. A prospective study of the value of monitoring heparin treatment with

Med 1972;287:324-7.
8 Raby C. Héparinothérapie contrólé. Presse Medicale 1967;75:1526

Yin ET, Wessler S, Butler J. Plasma heparin: a unique practical, submicrogramsensitive assay. If Lab Clin Med 1973;81:298-310.

Nelson $W$, Tong Y, Lee JK, Halberg F. Methods for cosinor rhythmometry. Chronobiologia 1979;6:305-23.

1 Brandt JT, Triplett DA. Laboratory monitoring of heparin. Effect of reagents and instruments on

12 Wessler S, Gitel SN. Heparin: new concepts relevant to clinical use. Blood $1979 ; 53: 525-44$

13 Kakkar VV, Djazaeri B, Fok J, et al. Low-molecular-weight heparin and prevention of postoperative deep vein thrombosis. Br Med $\mathcal{f} 1982 ; 284: 375-9$.

14 Reinberg A, Smolensky MH. Circadian changes of drug disposition in man. Clin Pharmacokinet 1982;7:401-20.

15 Petralito A, Mangiafico RA, Gibhno S, et al. Daily modifications of plasma fibrinogen, platelets aggregation, Howell's time, PTT, TT, and antithrombin 1982;9:195-201.

(Accepted 1 November 1984)

\section{YEARS AGO}

In recognition of public services rendered during the last epidemic of cholera, the French Government has recently awarded a large number of gold and silver medals, "mentions honorables," and "lettres de félicitations." In addition, a number of promotions in or to the Legion of Honour have been made. As is usual in such cases, the distribution of honours has been the cause of many heartburnings. Indignation-meetings have been held. Some complain that their merits have not been duly appreciated; others object to their names being associated with the names of others whom they consider to be adventurers and unworthy; while others again there are who decline their medals because they have not deserved them. At Montpellier, the dissatisfaction found expression in a noisy demonstration against the dean of the medical school, who had been made an officer of the Legion; and in favour of two other professors, who had shown special devotion during the epidemic, but had been rewarded only by medals. Before the uproar could be quelled, it was found necessary temporarily to close the medical school, and the dean has thought it prudent to resign. The minister who made the awards was a member of the Ferry cabinet, and resignation has, therefore, saved him from the consequences of his misguided generosity. (British Medical Fournal 1885;i:905.) . . . . .

.

